\title{
Treating Simple Tibia Fractures with Poly-DL-Lactic Acid Screw as a Locked Intramedullary Nail
}

\author{
Fang Dong*, Guang-Yi Li, De-Qiang Liu, An-Li Zhu and Ning Xu \\ Department of Orthopaedics, The Second People's Hospital of Liaocheng City, Liaocheng 252600, PR China
}

${ }^{*}$ For correspondence: Email: dong_lch@163.com; Tel: +86-0635-2342592

Received: 19 May 2015

Revised accepted: 27 September 2015

\begin{abstract}
Purpose: To investigate the curative effect of poly-DL-lactic acid (PDLLA) absorbable screw as a locked intramedullary nail for simple tibia fractures.

Methods: In this study, 35 patients treated with the PDLLA screw were observed, and another 35 patients treated with a traditional locking intramedullary nail were treated as controls. Intraoperative blood loss, postoperative complications, fracture-healing time, and Johner-Wruh grade at the last followup time point of the two treatment groups were compared.

Results: No patients experienced neurovascular injury during the operation, and no significant difference was observed in the operation and intraoperative blood loss of both two groups ( $p>0.05$ ). Postoperative $x$-ray results showed that the counterpoint and alignment of the fractures were favorable and that no rotational displacement had occurred. The follow-up period for all patients was $6-24$ months, with a mean of $15.6 \pm 6.7$ months. All patients treated with PDLLA screw were observed to have well-healed tibia fractures, while three patients in the traditional treatment group appeared to have undesirable fracture healing. No infection, breakage of the internal fixator, or rotational displacement was found in either of the two groups, and no significant difference was observed in complication incidence between the two groups ( $p>0.05)$. Additionally, Johner-Wruh grading showed no statistically significant difference between the two groups ( $p>0.05)$. Interestingly, the fracture-healing time of the PDLLA screw-treated patients was significantly shortened compared with that of traditional treatment patients $(p<0.05)$.

Conclusion: PDLLA screw, as a locked intramedullary nail, is a promising treatment for simple tibia fractures.
\end{abstract}

Keywords: Simple tibia fracture, Fracture healing time Poly-DL-lactic acid, Poly-DL-Lactic Acid, Absorbable screw, Dynamic stabilization, Internal fixator

Tropical Journal of Pharmaceutical Research is indexed by Science Citation Index (SciSearch), Scopus, International Pharmaceutical Abstract, Chemical Abstracts, Embase, Index Copernicus, EBSCO, African Index Medicus, JournalSeek, Journal Citation Reports/Science Edition, Directory of Open Access Journals (DOAJ), African Journal Online, Bioline International, Open-J-Gate and Pharmacy Abstracts

\section{INTRODUCTION}

It is well known that a tibia fracture is a common injury in traumatic orthopedics and is a serious bone injury seen in the clinic $[1,2]$. Therefore, an ideal treatment is crucial for the recovery of tibia fracture patients. Currently, with the use of small operative wounds, stable fixation, and strong anti-twisting force techniques, the intramedullary nail fixture is recognized as the first choice and a standard method for fixing backbone fractures of lower long bones [3-5].

However, the lock pin with interlocking intramedullary nail, which is commonly used in the clinic at present, is a metal material, which has strong stress shielding and easily leads to breakage of the lock pin $[1,3]$. In addition, 
sometimes there is a delay in union and nonunion when these metal materials are used $[6,7]$. The reasons for delayed union and nonunion are related to the degree of comminution, restoration, and incarceration of the soft tissue. In addition, the stress shielding of static locking is also an important factor that cannot be ignored during the fracture-healing process, and the stress shielding can be increased with the prolongation of fixation time, leading to lock pins that bend and break easily $[8,9]$.

Therefore, a strategy involving taking out the hemi-lock pin (dynamic fixation) is commonly adopted in the clinic when there is a tendency for delayed union [10,11]. However, this added surgery involves inconvenience and needless pain and financial burden for patients. Thus, there is an urgent need to find simple and effective strategies for treating tibia fractures.

In our present study, we designed a clinical investigation of the curative effects of the PolyDL-Lactic Acid (PDLLA) absorbable screw as a locked intramedullary nail for simple tibia fractures. From February 2010 to January 2014, 35 patients with simple tibia fractures were treated with the PDLLA screw as a distal locked intramedullary nail, and the treatment efficiency was satisfactory compared with the efficacy observed in 35 patients who were treated with traditional static fixation methods.

\section{EXPERIMENTAL}

\section{General data}

Our present study observed 70 patients in total (37 males and 33 females), ranging in age from 17 to 55 years. The patients were divided into two groups according to their sequence number at admittance to the hospital. Odd number patients constituted the treatment group (PDLLA screw treatment group), and even number patients constituted the control group (traditional treatment group). Both groups were treated with interlocking intramedullary screw fixation. The experimental group received the PDLLA absorbable screw instead of a distal locking intramedullary nail. The control group received a traditional metal lock pin with static fixation. All patients in the experimental group gave signed informed consent.

All patients had experienced a traumatic hemitibia closed fracture without a fibula fracture and without injury of the head, chest, and belly. Causes of injury were as follows: caused by traffic accident (51 patients), fall injury (16 patients), and wound delivered by another person (3 patients). The gender, age, wound, time of operation, position of fracture, and $\mathrm{AO}$ classification of the fracture showed no statistically significant differences between the two groups $(p>0.05)$ (Table 1). The study protocol was approved by the Ethics Committee of the Second People's Hospital of Liaocheng City (No. 20091108-2\#).

\section{Operation protocol}

Interlocking intramedullary nails used in this study were domestic 9-11-mm diameter and 260-340-mm length nails. The PDLLA screws used in our investigation were domestic $3.5-\mathrm{mm}$ external diameter and 25-35-mm length screws. Intramedullary nails of suitable length were chosen according to x-ray measurements and the length of the uninjured side tibia prior to the operation.

Under continuous epidural anesthesia, a 4-5-cm incision was made on the front middle ligamentum patellae, then the tubercle of the tibia above was revealed by cutting part of the ligamentum patellae, and the mouth gag trepanned on the upward side of the tibia tubercle. After that, closed reduction was carried out. After opening the medullary canal, according to the reaming diameter (less than $1 \mathrm{~mm}$ ), suitable intramedullary nails were chosen for insertion into the marrow cavity.

After confirming the correctness of the counterpoint and alignment of the fracture by $x$ ray,

Table 1: Baseline demographics and disease characteristics

\begin{tabular}{|c|c|c|c|c|c|c|c|c|c|c|}
\hline \multirow{2}{*}{ Group } & \multirow{2}{*}{$\mathbf{N}$} & \multicolumn{3}{|c|}{ Gender } & \multirow{2}{*}{$\begin{array}{l}\text { Wounded } \\
\text { time } \\
\text { operation } \\
\text { (day) }\end{array}$} & \multicolumn{3}{|c|}{ Position of fracture } & \multicolumn{2}{|c|}{$\begin{array}{c}\text { AO } \\
\text { classification }\end{array}$} \\
\hline & & $\mathbf{M}$ & $\mathbf{F}$ & Age & & Oberhalb & Midpiece & Hypomere & A & B \\
\hline Treatment & 35 & 19 & 16 & $36.6 \pm 9.6$ & $2.2 \pm 0.7$ & 7 & 12 & 16 & 12 & 23 \\
\hline Control & 35 & 18 & 17 & $37.1 \pm 9.8$ & $2.1 \pm 0.8$ & 6 & 15 & 14 & 14 & 21 \\
\hline$p$-values & - & \multicolumn{2}{|c|}{0.811} & 0.835 & 0.872 & & 0.762 & & \multicolumn{2}{|c|}{0.621} \\
\hline
\end{tabular}


patients in the treatment group were locked in with two PDLLA screws in the far end of the intramedullary nails and locked in the metal lock pin in near end. For the control group, patients were locked in with regular metal lock pin in both ends of the intramedullary nails.

\section{Postoperative treatment}

For all patients, antibiotics were applied until $24 \mathrm{~h}$ after surgery. On the second day after surgery, patients began to do limb training without loads in bed, avoiding rotation of the knee and ankle. Patients could walk with a load and with the aid of crutches at six weeks after the operation. Xrays were checked regularly each month until fracture healing without crutches and entire loadbearing.

\section{Observation index}

In our present study, we compared the following indexes between the treatment and control groups: time of operation, blood loss value during operation, postoperative complications (including infection, breakage of the internal fixator, rotation displacement, delayed union of the tibia, nonunion, etc.), fracture-healing time, and the Johner-Wruh grade at the last follow-up.

\section{Statistical analysis}

Data are expressed as mean \pm SD, and SPSS 19.0 statistical software was used in this study. The t-test was applied to evaluate the mean value of two independent samples, and enumeration data were analyzed by $\mathrm{X}^{2}$ test.
Differences were considered significant at $p<$ 0.05 .

\section{RESULTS}

As can be seen from Table 2, all patients in both the treatment and control groups had no injury of vessels or nerves during the operation, and the operation time and periwig-operative bleeding were not statistically different between the two groups $(p>0.05)$. A post-operation $x$-ray showed that the counterpoint and alignment of the fractures were favorable without rotation displacement. All patients underwent follow-up evaluations that lasted 6-24 months, and the average time was $15.6 \pm 6.7$ months. By treating with PDLLA screw, all patients' tibia fractures healed well, whereas three patients in the control group demonstrated undesirable fracture healing (delayed union) and finally healed by hemi-lock pin motorization. No complications, including infections, breakage of the internal fixator, or rotational displacement were observed in the two groups, and no significant difference was observed in complication incidence between the two groups ( $p>0.05$, Table 2). Additionally, results of Johner-Wruh grading showed no statistically significant difference between the two groups ( $p>0.05$, Table 2$)$. Interestingly, fracturehealing time of the PDLLA screw-treated patients was significantly shortened compared with the traditional treatment patients ( $p<0.05$, Table 2$)$. Figure 1 shows the results of an $x$-ray from typical cases before and after operation in the treatment groups.

Table 2: Operation results of the control and treatment groups

\begin{tabular}{|c|c|c|c|c|c|c|c|}
\hline \multirow[b]{2}{*}{ Group } & \multirow[b]{2}{*}{$\mathbf{N}$} & \multirow{2}{*}{$\begin{array}{l}\text { Operation } \\
\text { time (min) }\end{array}$} & \multirow{2}{*}{$\begin{array}{l}\text { Blood loss } \\
\quad(\mathrm{ml})\end{array}$} & \multirow{2}{*}{$\begin{array}{l}\text { Fracture healing } \\
\text { time (week) }\end{array}$} & \multirow{2}{*}{$\begin{array}{l}\text { Complication } \\
\text { Incidence (\%) }\end{array}$} & \multicolumn{2}{|c|}{ Ohner-Wruh grade } \\
\hline & & & & & & Superior & Fine \\
\hline Treatment & 35 & $42.3 \pm 9.0$ & $97.3 \pm 11.3$ & $14.2 \pm 0.9$ & $0(0 / 35)$ & 29 & 6 \\
\hline Control & 35 & $45.1 \pm 10.0$ & $95.3 \pm 9.1$ & $15.3 \pm 0.7$ & $3(3 / 35)$ & 27 & 8 \\
\hline$P$ value & - & 0.215 & 0.418 & 0.000 & 0.248 & 0.55 & \\
\hline
\end{tabular}
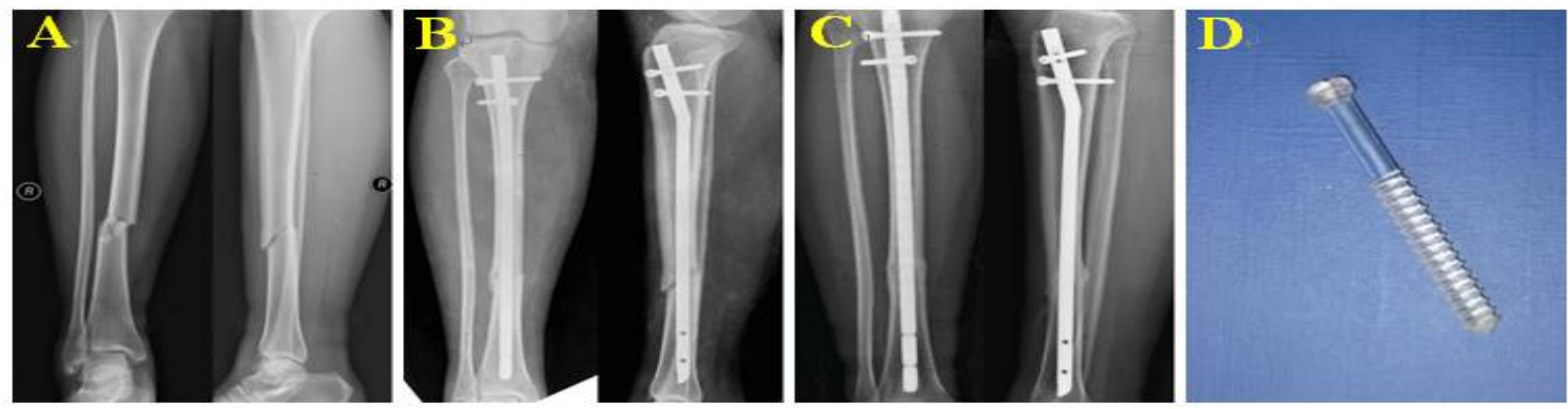

Figure 1: Results of x-ray determination. Female patient, 32 years, 1/3 fracture of simple tibia below the average. (A). Preoperative X-ray showed malposition of broken ends; (B). Counterpoint and alignment of fracture were well healed after treating with PDLLA absorbable screw as locked intramedullary nail; (C). Fracture line disappeared and fracture healed well at 14 weeks after operation; (D). PDLLA absorbable screw used in our present study 


\section{DISCUSSION}

Based this clinical comparative investigation, there is no obvious difference between the therapeutic results for simple tibia fracture between the PDLLA screw treatment and traditional treatment. However, treatment with the PDLLA screw can achieve a shorter fracturehealing time than traditional treatment. Therefore, our present study found that it is effective to use the PDLLA absorbable screw as an interlocking intramedullary distal lock pin to fix simple tibia fractures.

The PDLLA absorbable screw is a safe and transparent non-crystallizable polymer, and it has favorable histocompatibility. In addition, it swells 24-48 $\mathrm{h}$ after imbedding within the body of patient, and can enhance soundness of internal fixation and sclerosis $[12,13]$. The initial bending strength of the PDLLA screw is greater than 130 $\mathrm{Mpa}$, and the tensile strength is greater than 48 Mpa. Moreover, the elasticity modulus of the PDLLA screw is between 2.0 to $3.0 \mathrm{Gpa}$, which is not as firm as a metal lock pin, but may satisfy the need of joint functional training without the burden of the intramedullary lock pin.

The mechanical strength of the PDLLA screw is lost gradually after 12 weeks in the imbedding body. PDLLA is first degraded to lactic acid in body and then changed into carbon dioxide and water via the tricarboxylic acid cycle [14,15]. Previous reports also indicate that the initial stage of the tibia that distal locked by the PDLLA screw is static fixation, and the mechanical strength loss time is identical with the time of motorization, which is generally $6-12$ weeks. Finally, the PDLLA screw is absorbed by degradation or disintegration [16]. In this way, it benefits fracture healing, avoiding the risk and embarrassment of the metal lock pin breaking down. Furthermore, it does not have the disadvantages associated with the traditional screw, which needs to be taken out. Therefore, it reduces patient pain and inconvenience. Collectively, treatment with the PDLLA absorbable screw instead of a traditional metal lock pin is associated with many benefits $[14,17,18]$ : (i) patients do not need to suffer the removal surgery of the metal implants; (ii) patients do not need to take out hemi-lock pin in the treatment process; and (iii) the PDLLA absorbable screw possesses an elastic modulus almost equal to that of cancellous bone, thus it is good for callus formation and fracture healing.

In our present study, we used the PDLLA absorbable screw to treat simple tibia fractures based on the following reasons: (i) simple tibia fractures are commonly the relative simple $A$ and $B$ type fractures, and can generally achieve relative stability after restoration; (ii) the thick intramedullary nail can be used after being reamed, and the friction force between the nail and bone wall control the fracture rotation to some extent; and (iii) the fibula, which can support $1 / 6$ of the whole body, forms a firm and integrated mechanical structure via connecting with the tibia through up and down tibiofibular syndesmosis, thus supporting the normal force line and length of the shank and efficiency controlling the rotation of tibia fracture.

In addition, the reasons why we did not chose the distal lock pin are: (i) the far end is the weakest intramedullary nail fixation and the lock pin could easily break up; (ii) no suitable proximal length of the PDLLA screw could be selected at the present time; and (iii) intramedullary nail motorization can easily cause an increase of the end of the nail, leading to a disadvantage for the functional recovery of the knee-joint [19].

\section{Limitations of the study}

In the study, there were only 35 patients, and therefore more samples are needed to evaluate the curative effects of PDLLA screw as locked intramedullary nail in a future study.

\section{CONCLUSION}

Based on the results obtained in this study and those of others, the following rules need to be considered when applying the PDLLA screw for the treatment of a simple tibia fracture: (i) tapping is needed during the operation to penetrate the bilateral cortex of the bone due to the weak antitwisting of the PDLLA screw; otherwise, it is easy to cause lock breakage; (ii) local friction easily leads to inflammation when the PDLLA screw subcutaneously embeds too shallowly, and thus it is better to use a countersunk instrument to sink the nail head into the bone cortex; and (iii) patients should gradually accumulate weight burden on the hurt limb and get $x$-rays checked regularly.

\section{REFERENCES}

1. Johner R, Wruh O. Classification of tibial shaft fractures sand correlation with results after tigid fixation. Clin Orthop 1995; 315: 56-63.

2. Makhdoom A, Maheshwari LD, Laghari MA, Tahir SM, Ali $S M$, Siddiqui KA. Open proximal \& distal fractures of tibia treated with Naseer Awais External Fixator with T-clamp. J Pak Med Assoc. 2015; 65:727-732. 
3. Wang SW, Wu HX, Cui JJ. Meta-analysis of curative and security on treating with interlocking intramedullary nail and compression plate. Chin Clin Physic J 2014; 20: 3646-3652.

4. Wilkey KD, Mehserle W. Mechanical characteristics of eight femoral intramedullary nailing systems. J Orthop Trauma 1998; 12:177-185.

5. Arlettaz Y, Dominguez A, Farron A, Ehlinger M, Moor BK. Distal locking of femoral nails: evaluation of a new radiation-independent targeting system. J Orthop Trauma. 2012; 26:633-637.

6. Höntzsch $D$, Schaser KD, Hofmann GO, Pohlemann $T$, Hem ES, Rothenbach E, Krettek C, Attal R. Evaluation of the effectiveness of the angular stable locking system in patients with distal tibial fractures treated with intramedullary nailing: a multicenter randomized controlled trial. J Bone Joint Surg 2014; 96: 1889-1897.

7. Ke SZ, Yang RY, Luo Y. Treating 1/3 fracture below the average of tibiofibula with interlocking intramedullary combined steel plate. Clin Orthoped J 2013; 16: 6567.

8. Yan XG, Bao TZ, Zhao WD. Biomechanical property of thighbone static interlocking intramedullary nail and influence on fracture healing. Chin J Tissue Engine Res 2010; 14:3123-3126.

9. Min J, He SJ, Zheng $H$. Studying on the curative efficiency of treating fracture of lower limb with motorize interlocking intramedullary nail in early stage. Chongqing Med 2013; 25: 2988-2990.

10. Liu Y, Chen $Q Q$, Hou CL, Gou SH, Chen AM, Ouyang $Y P$, Kuang $Y$. Treatment of femoral shaft comminuted fractures with interlocking intramedullary nail. Chin J Orthop Trauma 2004; 6: 382-385.

11. Hente $R$, Cordey J, Rahn BA, Maghsudi $M$, von Gumppenberg S, Perren SM. Fracture healing of the sheep tibia treated using a unilateral external fixator.
Comparison of static and dynamic fixation. Injury 1999; 30 Suppl 1:A44-51.

12. Achtnich A1, Forkel P, Metzlaff S, Zantop T, Petersen W. Degradation of poly-D-L-lactide (PDLLA) interference screws (Megafix®). Arch Orthop Trauma Surg 2014; 134:1147-1153.

13. Richmond J, Colleran K, Borens O. Nonunions of the distal tibia treated by reamed intramedullary nailing. $J$ Orthop Trauma 2004; 18: 603-610.

14. Tang J, Hu JF, Guo WC, Yu L, Zhao SH. Research and application of absorbable screw in orthopedics: a clinical review comparing PDLLA screw with metal screw in patients with simple medial malleolus fracture. Chin J Traumatol. 2013;16:27-30.

15. Hu YL, Yuan WQ, Wang LF, Liu HF, Jin D. A prospective randomized trial of poly-DL-lactic acid absorbable and metallic screws for treatment of syndesmotic disruptions. Nan Fang Yi Ke Da Xue Xue Bao 2010; 30:2360-2362.

16. Egol K A, Weisz R, Hiebert R, Tejwani NC, Koval KJ, Sanders RW. Does fibular plating improve alignment after intramedullary nailing of distal metaphyseal tibia fractures? J Orthop Trauma 2006; 20: 94-103.

17. Rasse M, Moser D, Zahl C, Gerlach KL, Eckelt U, Loukota R. Resorbable poly (D, L) lactide plates and screws for osteosynthesis of condylar neck fractures in sheep. Br J Oral Maxillofac Surg 2007;45:35-40.

18. Macarini L, Murrone M, Marini S, Mocci A, Ettorre GC. $M R I$ in ACL reconstructive surgery with PDLLA bioabsorbable interference screws: evaluation of degradation and osteointegration processes of bioabsorbable screws. Radiol Med 2004; 107:47-57.

19. Zhang F, Luo XD, Wang ZZ. Studying on the comparison between motorization and no motorization which being done after treating tibia shaft fracture with interlocking intramedullary nail. Chongqing Med 2013; 29: 3491-3495. 